\title{
Fractional Quantum Hall States with Non-Abelian Statistics
}

\author{
G. MÖlleR ${ }^{a}$, A. WóJS ${ }^{a, b}$ AND N.R. COOPER ${ }^{a}$ \\ ${ }^{a}$ TCM Group, Cavendish Laboratory, University of Cambridge, Cambridge, UK \\ ${ }^{b}$ Institute of Physics, Wrocław University of Technology, Wrocław, Poland
}

\begin{abstract}
Using exact numerical diagonalization we have studied correlated many-electron ground states in a partially filled second Landau level. We consider filling fractions $\nu=1 / 2$ and $2 / 5$, for which incompressible quantum liquids with non-Abelian anion statistics have been proposed. Our calculations include finite layer width, Landau level mixing and arbitrary deformation of the interaction pseudopotential. Computed energies, gaps, and correlation functions support the non-Abelian ground states at both $\nu=1 / 2$ ("Pfaffian") and $\nu=2 / 5$ ("parafermion" state).
\end{abstract}

PACS numbers: 73.43.-f, 71.10.Pm

\section{Introduction}

Fractional quantum Hall systems, especially some of the incompressible quantum liquids in the second Landau level $\left(\mathrm{LL}_{1}\right)$ realized recently in the high-mobility GaAs quantum wells [1], are the most promising candidates for the physical realization of hypothetical non-Abelian anion quantum statistics in two dimensions (2D) [2]. The idea of non-Abelian statistics has recently attracted renewed interest in the context of fault protected topological quantum computation [3].

One well-known non-Abelian ground state is the Moore-Read (MR) "Pfaffian" wave function [4, 5] for a half-filled $\mathrm{LL}_{1}$ (filling fraction $\nu=1 / 2$ ). It corresponds to a 2D $p$-wave superconductor of spinless composite fermions (CFs). A couple of other candidates, both proposed for $\nu=2 / 5$, are the "parafermion" state of Read and Rezayi (RR) [6] corresponding to triplets of particles and the paired Bonderson-Slingerland (BS) state [7].

Existing exact diagonalization studies point to the MR state as the only likely incompressible quantum liquid at $\nu=1 / 2[5]$. So, the experimentally observed incompressibility is a convincing argument in favor of the physical realization of the (non-Abelian) MR wave function. Still, the apparent sensitivity of the actual $\nu=1 / 2$ ground state in $\mathrm{LL}_{1}$ to the finite layer width or LL mixing, as well as the recently reported [1] discrepancy between the measured and computed excitation energy gaps at $\nu=1 / 2$ in $\mathrm{LL}_{1}$, both invite further studies. The situation at $\nu=2 / 5$ in $\mathrm{LL}_{1}$ is even less clear, as the two adiabatically disconnected RR and BS states (with distinct braiding properties) compete with each other and also with the Jain wave function of non-interacting CFs, known from LL [6]. It remains crucial to establish if the actual $\nu=2 / 5$ ground state in $\mathrm{LL}_{1}$ is non-Abelian (and, if so, of what kind).

Here, we have used exact diagonalization in the search of non-Abelian states in $\mathrm{LL}_{1}$. Indeed, from the analysis of computed energies, excitation gaps, and correlation functions, we argue that the MR and RR non-Abelian states should emerge at the appropriate fillings of $\mathrm{LL}_{1}$.

\section{Results and discussion}

In order to allow for the expected sensitivity to (e.g.) the layer width $w$, we began by modeling a general short-range repulsive interaction by a Haldane pseudopotential with arbitrary three leading coefficients $U_{1}, U_{3}, U_{5}$ (and $U_{m>5}=0$ ), and studied the phase diagram at various filling factors $\nu$. The calculations were carried out on a sphere, where the LL degeneracy is determined by the single-particle angular momentum $l$, and different (universality classes of) ground states are quite conveniently distinguished by different "shifts" $\gamma$ defined by the relation: $2 l=N / \nu-\gamma$. For such a series of $N$-electron states to represent an incompressible liquid, the ground states should be consistently non-degenerate (total angular momentum $L=0$ for each $N$ ) and the excitation gap $\Delta$ should be significant and persist at $N \rightarrow \infty$.
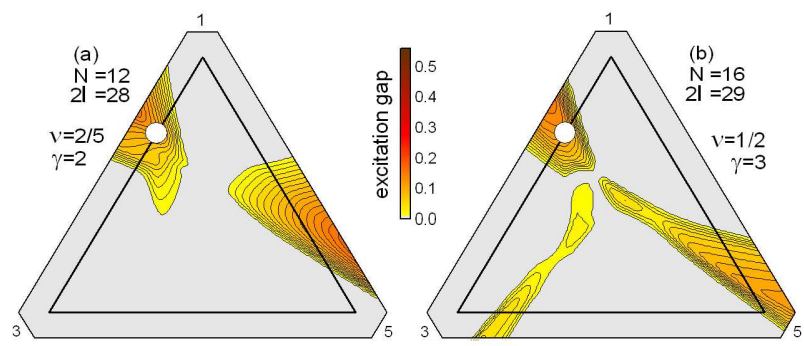

Fig. 1. Ternary contour plots of neutral excitation gaps $\Delta$ for $N$ fermions in a partially filled Landau level with shell angular momentum $l$, interacting through $U_{m}$ (corners of the big triangles correspond to $U_{m}=\delta_{m, \mu}$ with $\mu=1,3$, and 5 , as marked). White circles mark positions of $\mathrm{LL}_{1}$.

The maps of $\Delta$ shown in Fig. 1, and similar (omitted) maps of the overlap with the MR state (for $\nu=1 / 2$ ) and of the various relevant two- and three-body correlation coefficients demonstrate (i) the adiabatic connection of the $\nu=1 / 2$ ground state to the MR liquid characterized by $\gamma=3$, and (ii) the emergence of a $\nu=2 / 5$ ground state with the BS-like $\gamma=2$ for a similar range 
of interactions as those yielding the MR state. While, for the lack of competing series of gapped $L=0$ states with a different $\gamma$, this settles the situation for $\nu=1 / 2$, competition between the BS, RR, and Jain states at $\nu=2 / 5(\gamma=2,-2$, and 4 , respectively $)$ must still be resolved. This is done by the comparison of extrapolated correlation energies per particle, $E$, converted from the $N$-electron energies obtained from exact diagonalization in a standard way [8], by including charge-compensating background and aligning the energy units $e^{2} / \lambda(\lambda$ being the magnetic length) to become independent of $\gamma$.

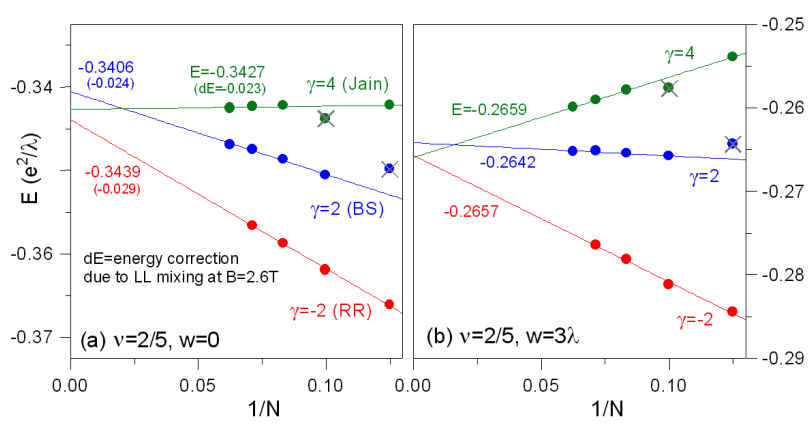

Fig. 2. Correlation energy $E$ as a function of electron number $N$ in the $L_{1}$, for the competing series of Jain, $\mathrm{BS}$, and RR ground states at $\nu=2 / 5$, for layer widths $w=0$ and $3 \lambda$. Crossed out points were excluded from extrapolation (size effects). In (a), $\mathrm{d} E$ are the energy corrections due to LL mixing, calculated at $B=2.6 \mathrm{~T}$.

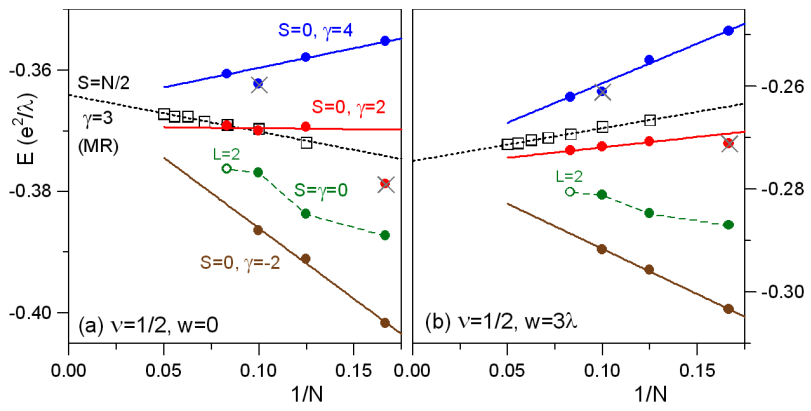

Fig. 3. Correlation energies $E$ of several unpolarized $(S=0)$ ground states with even $\gamma$ and of the polarized $(S=N / 2)$ states with $\gamma=3$ (MR class), all for $\nu=1 / 2$, $\mathrm{LL}_{1}, w=0$ and $3 \lambda$. Crossed out points were excluded from extrapolation (states aliased with $\nu=2 / 3$ and $\gamma=-1)$.

The energies $E$ of the BS, RR, and Jain states at $\nu=2 / 5$ are shown in Fig. 2, for both $w=0$ and $3 \lambda$ (a reasonable experimental value). Since all states extrapolate to nearly the same energy, the ground state in a real system is likely to be determined by the factors ignored in the present calculation - probably by different susceptibility to the LL mixing in a finite magnetic field $B$. We have estimated the appropriate reduction $\mathrm{d} E$ by additionally including in the diagonalization of the $N$-electron states with one cyclotron excitation. Although such basis expansion strongly limits $N$ for which the diagonalization can be performed, we found that $\mathrm{d} E$ is far less $N$-dependent than $E$, and used the values obtained for $N \leq 10$ (marked in Fig. 2). While $B=2.6 \mathrm{~T}$ is a rather weak field, in which the present approximate treatment of the LL mixing is probably insufficient, the tendency is fairly obvious: it is the "parafermion" $\mathrm{RR}$ state whose energy $E$ is reduced most strongly by LL mixing, making it a well resolved ground state in realistic experimental conditions.

Finally, we have studied spin polarization at $\nu=1 / 2$ in $L_{1}$. In Fig. 3 we plot the correlation energies $E$ of the spin-unpolarized $(S=0)$ series with even shifts $\gamma$ (at odd shifts the ground states are degenerate). From the limited data $(N \leq 12)$, we find that only those $S=0$ states with $\gamma=\overline{2}$ and 4 might possibly have lower $E$ than the MR state (also shown) for large $N$. However, comparison of pair correlation functions [8] reveals that both these $S=0$ states are the topological spin textures (skyrmions) formed over the polarized MR state rather than genuine unpolarized ground states with different correlations (and lower energy $E$ ).

\section{Conclusion}

We have presented numerical evidence that both fractional quantum Hall states observed in $\mathrm{LL}_{1}$ at $\nu=1 / 2$ and $2 / 5$ are adiabatically connected to the non-Abelian (MR and RR) wave functions. For $\nu=2 / 5$, the competition with the Abelian Jain state (and another non-Abelian BS state) is close, but it seems that it is resolved in favor of the RR state by the LL mixing in a finite magnetic field.

\section{Acknowledgments}

The authors thank S.H. Simon for insightful discussions. A.W. was supported by Marie Curie Intra-European Grant PIEF-GA-2008-221701.

\section{References}

[1] C.R. Dean, B.A. Piot, P. Hayden, S. Das Sarma, G. Gervais, L.N. Pfeiffer, K.W. West, Phys. Rev. Lett. 100, 146803 (2008).

[2] C. Nayak, S.H. Simon, A. Stern, M. Freedman, S. Das Sarma, Rev. Mod. Phys. 80, 1083 (2008).

[3] A.Yu. Kitaev, Ann. Phys. (New York) 303, 1 (2003).

[4] G. Moore, N. Read, Nucl. Phys. B 360, 362 (1991).

[5] G. Möller, S.H. Simon, Phys. Rev. B 77, 075319 (2008).

[6] N. Read, E. Rezayi, Phys. Rev. B 59, 8084 (1999).

[7] P. Bonderson, J.K. Slingerland, Phys. Rev. B 78, 125323 (2008).

[8] R.H. Morf, Phys. Rev. Lett. 80, 1505 (1998). 